\title{
Desempeño fisiológico de nueve genotipos de cacao (Theobroma cacao L.) bajo la sombra de tres especies forestales en Santander, Colombia
}

\section{Physiological performance of nine cacao (Theobroma cacao L.) genotypes under the shadow of three forest species in Santander, Colombia}
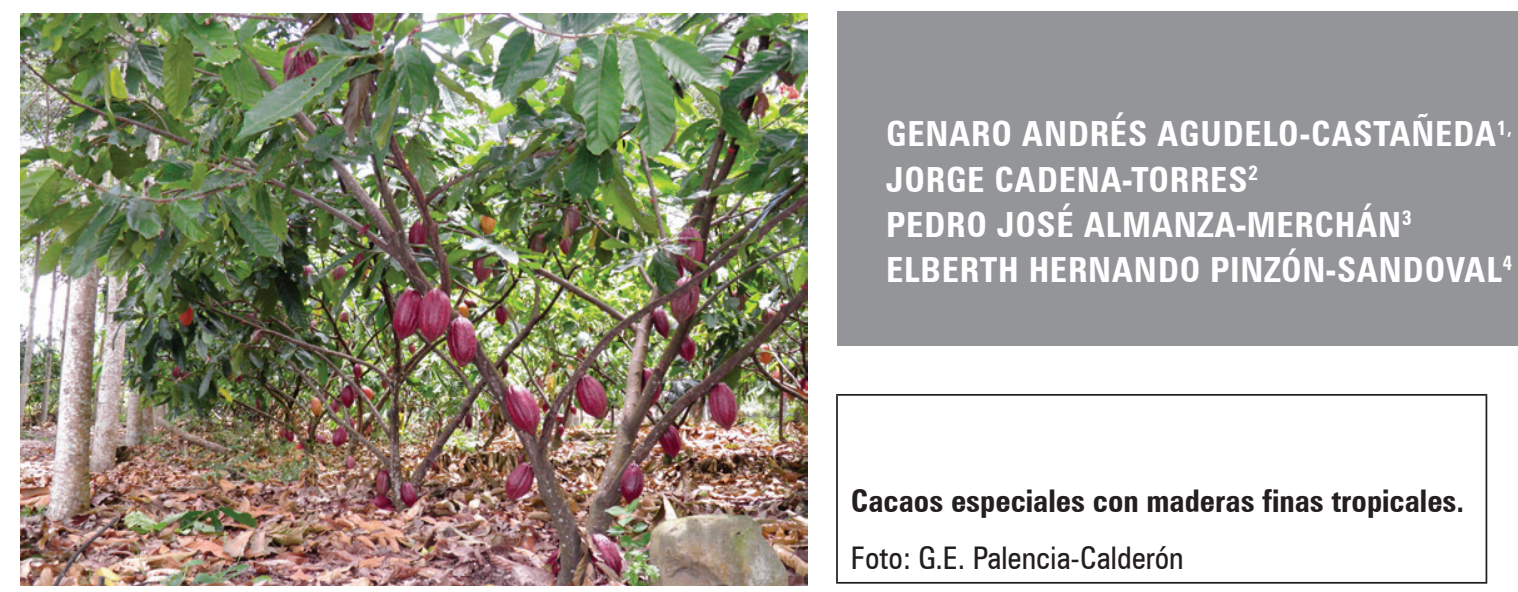

\section{RESUMEN}

En la actualidad, Colombia tiene 173.000 ha cultivadas con Theobroma cacao, de las cuales la mayoría están establecidas en asocio con árboles frutales o maderables, además de proveer sombra pueden generar ingresos para el cacaocultor. Para seleccionar especies que conforman el arreglo agroforestal, es necesario considerar los requerimientos ecofisiológicos de los genotipos de cacao y la interacción con su entorno agroecológico, incluido el sombrío. El objetivo de esta investigación fue evaluar el rendimiento fisiológico de nueve clones de cacao establecidos en sistema agroforestal con Cariniana pyriformis, Hevea brasiliensis en El Carmen de Chucurí y C. pyriformis, Tectona grandis en Rionegro, Santander. Se realizó seguimiento a la tasa de fotosíntesis, transpiración, conductancia estomática y uso eficiente del agua, tanto en la época húmeda, como en la seca. Los resultados mostraron que los sistemas de sombrío influyen sobre las tasas de fotosíntesis que presentan las plantas de cacao. Estos clones establecidos bajo el sistema de sombrío con C. pyriformis, presentan mayores tasas de fotosíntesis $\left(5,39 \mu\right.$ moles $\left.\mathrm{CO}_{2} \mathrm{~m}^{-2} \mathrm{~s}^{-1}\right)$, en la época húmeda y seca. Lo anterior se atribuyó al tipo de crecimiento y sombra proveída por esta especie, que ofrece mejores condiciones ambientales para el desempeño fisiológico del cacao. Se recomienda esta especie forestal como sombrío, para el establecimiento de nuevas

1 Centro de Investigación La Suiza, Corporación Colombiana de Investigación Agropecuaria (Corpoica), Rionegro (Colombia). ORCID Agudelo-Castañeda, G.A.: 0000-0002-0469-1406

2 Centro de Investigación Turipaná, Corporación Colombiana de Investigación Agropecuaria (Corpoica), Cereté (Colombia). ORCID Cadena-Torres, J.: 0000-0002-5180-2893

3 Facultad de Ciencias Agropecuarias, Grupo de Investigaciones Agrícolas (GIA), Universidad Pedagógica y Tecnológica de Colombia (UPTC), Tunja (Colombia). ORCID Almanza-Merchán, P.J.: 0000-0002-9207-0617

4 Facultad de Ciencias Agropecuarias, Universidad Pedagógica y Tecnológica de Colombia (UPTC), Tunja (Colombia). ORCID Pinzón-Sandoval, E.H.: 0000-0001-9229-3450

5 Autor para correspondencia. gagudelo@corpoica.org.co 
áreas de cacao en el departamento de Santander. Individualmente, los clones de cacao con el mejor comportamiento ecofisiológico fueron TCS19 y TCS13, por lo que se constituyen en genotipos de importancia agronómica.

Palabras clave adicionales: agroforestería, cultivares de cacao, producción de cultivos,
Cariniana pyriformis, variación estacional, respuesta fisiológica.

\section{ABSTRACT}

At present, Colombia has 173,000 ha cultivated with Theobroma cacao, most of which are established in association with fruit or timber trees that provide shade, that can generate income for cocoa growers. In order to select species that make up the agroforestry system, it is necessary to consider the ecophysiological requirements of T. cacao genotypes and the interaction with the agroecological environment, including shade. The objective of this research was to evaluate the physiological performance of nine cocoa clones established in an agroforestry system with Cariniana piryformis, Hevea brasiliensis (Carmen) Cariniana piryformis, and Tectona grandis (Rionegro, Santander province). The photosynthesis rates, transpiration, stomatal conductance and efficient use of water in cocoa clones was monitored in the wet and dry season. The results showed that shade systems influence the photosynthetic rates of cocoa plants. The clones established under the shade system with C. piryformis presented higher rates of photosynthesis (5.39 $\mu$ moles $\left.\mathrm{CO}^{2} \mathrm{~m}^{-2} \mathrm{~s}^{-1}\right)$, both in the wet season and in the dry season. This was attributed to the type of growth and shade provided by this species, which offers better environmental conditions for the physiological performance of cocoa. As a result, this forest species is recommended for shading in the establishment of new areas of $T$. cacao in the Department of Santander. Individually, the cocoa clones with better echophysiological behavior included TCS19 and TCS13, making their genotypes agronomically important.

Additional key words: agroforestry, cocoa cultivars, crop production, Cariniana pyriformis, seasonal variation physiological response.

Fecha de recepción: 01-09-2017 Aprobado para publicación: 30-01-2018

INTRODUCCIÓN

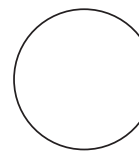

El cacao (Theobroma cacao L.) se ha convertido en un reglón de importancia agrícola en diferentes países, debido a sus múltiples usos, como la elaboración de chocolates y productos derivados (Jaimez et al., 2008). En Colombia, el área sembrada está alrededor de 173.000 ha con una producción de $56.785 \mathrm{t}_{\text {año }}{ }^{-1}$ e grano seco. Santander es el primer productor con el $38,9 \%$ de la producción nacional, seguido por Arauca con $11,3 \%$ y Antioquia con 9,3\% (Fedecacao, 2017). Las siembras en Colombia se han realizado con materiales híbridos trinitarios y amazónicos, descendientes de híbridos y clones introducidos (García, 2014). Por tal razón, se cuenta con diversidad genética adaptada a los parámetros climáticos diversos, que se presentan en las zonas agroecológicas donde se cultiva (Almeida y Valle, 2007). Theobroma cacao por su hábito de crecimiento, se ha sembrado en asocio con especies como frijol (Phaseolus vulgaris L.), yuca (Manihot esculenta Crantz), plátano (Musa sp.) como sombríos transitorios; mientras frutales y maderables como sombríos permanentes, los cuales además de proveer sombra le permiten al agricultor tener otras alternativas de ingreso que inicie la producción de T. cacao (Mejía y Palencia, 2004). Corpoica, desde el año 1998 ha colectado, evaluado y seleccionado materiales genéticos de T. cacao en fincas de productores, los cuales se vincularon a las colecciones Selección Colombia (SC) y Selección Colombia Corpoica (SCC) (Arguello et al., 1999). A partir de estos, se seleccionaron ocho genotipos y un testigo para evaluaciones regionales, los cuales se establecieron en modelos agroforestales con maderas finas tropicales, partiendo del hecho de 
que la planta de T. cacao no es tolerante a niveles altos de radiación (Jaimez et al., 2008). En Colombia son escasas las investigaciones que permitan entender las relaciones ecofisiológicas que se establecen entre especies de los sistemas agroforestales. En esta investigación se estudió el comportamiento ecofisiológico de nueve clones de T. cacao, establecidos en sistemas agroforestales en combinación con dos especies forestales en los municipios de El Carmen de Chucurí y Rionegro, Santander. Lo anterior con el fin de evaluar el desempeño fisiológico de los clones, bajo los sistemas de sombrío.

\section{MATERIALES Y MÉTODOS}

Para el desarrollo de esta investigación se utilizaron plantaciones de T. cacao establecidas en el año 2008 en dos localidades del departamento de Santander (Colombia). La primera en el municipio de El Carmen de Chucurí ubicada a $298 \mathrm{msnm}$, temperatura promedio del aire $30^{\circ} \mathrm{C}$, precipitación media anual de $1.800 \mathrm{~mm}$ y luminosidad máxima de $2.100 \mu$ moles $\mathrm{m}^{-2} \mathrm{~s}^{-1} \mathrm{de}$ fotones; la segunda localidad en el municipio de Rionegro en el centro de investigación La Suiza con una altitud de $550 \mathrm{msnm}$, temperatura promedio del aire $25^{\circ} \mathrm{C}$, precipitación media anual mayor a $2.000 \mathrm{~mm}$ y luminosidad máxima de $1.800 \mu$ moles $\mathrm{m}^{-2} \mathrm{~s}^{-1}$ de fotones. Estos datos fueron registrados mediante estaciones meteorológicas establecidas en cada localidad (Watchdog serie 2000, Spectrum Technologies Inc, Aurora, IL, USA). En Rionegro, los clones contaban con sombrío constituido por árboles de abarco (Cariniana pyriformis Miers) y teca (Tectona grandis L.f.) con alturas de $12 \mathrm{~m}$ en promedio a una densidad de 340 árboles/ha; en El Carmen, los clones contaban con sombrío constituido por árboles de abarco y caucho (Hevea brasiliensis (Willd. ex A.Juss.) Müll.Arg.) con alturas promedio de $14 \mathrm{~m}$ y densidad de 340 árboles/ ha. Los clones regionales de cacao evaluados fueron: Theobroma Corpoica La Suiza (TCS) 13, 19; Selección Colombia Corpoica (SCC) 53, 82, 83; Colección Corpoica La Suiza (CCS) 73, 77, 80 e Imperial College Selection (ICS) 95, establecidos a $3 \times 3 \mathrm{~m}$ para una densidad de 900 plantas/ha. Las evaluaciones de fotosíntesis se realizaron durante el 2016 en plantaciones de cacao de ocho años de edad, en plena producción.

Se realizaron mediciones ecofisiológicas a los clones, registrando la radiación fotosintéticamente activa $(P A R)$, tasa de fotosíntesis neta $(A)$, tasa de transpiración $(E)$, conductancia estomática $\left(g_{s}\right)$ y eficiencia instantánea en el uso del agua (EUA), mediante el uso de un equipo portátil de intercambio gaseoso (ADC Lc Pro, Hoddesdon, UK). Se realizaron mediciones en época húmeda y seca, correspondientes a épocas de mayor y menor intensidad de lluvia. Para las medidas de intercambio gaseoso se utilizó la cuarta o quinta hoja de la rama localizada en el tercio medio del dosel del árbol. Las mediciones se realizaron entre las 08:00 y las 12:00 h, bajo condiciones de la radiación natural para cada sistema agroforestal, conservando la posición natural de la hoja con respecto a la radiación incidente.

Se utilizó un diseño de bloques completos al azar con 18 tratamientos correspondientes al arreglo de nueve clones de cacao bajo dos sistemas de sombrío y dos épocas del año, en un arreglo factorial $9 \times 2 \times 2$. Con los resultados obtenidos se realizaron pruebas de normalidad y homogeneidad de varianzas, análisis de varianza (ANOVA) para determinar diferencias entre tratamientos $(P \leq 0,05)$. En caso de detectar diferencias estadísticas, se realizaron pruebas de comparación de medias utilizando la prueba HSD de Tukey. El análisis de los datos se realizó utilizando el paquete estadístico SAS v. 9.3. En total se realizaron cuatro mediciones, dos en época seca y dos en época húmeda. Para la medición se utilizó un flujo entre 197,3 y 201,7 $\mu$ moles s ${ }^{-1}$, a la concentración ambiental de $\mathrm{CO}_{2}$ $\left(381 \mathrm{~g} \mathrm{~m}^{-3}\right)$.

\section{RESULTADOS Y DISCUSIÓN}

Las mediciones se realizaron en dos épocas, una de mayor precipitación (época húmeda) y otra de menor precipitación (época seca). Al momento de la medición se tomaron muestras con el fin de determinar la humedad actual del suelo, las cuales se secaron posteriormente en horno mufla a $70^{\circ} \mathrm{C}$ para determinar la humedad por diferencia de pesos. En la tabla 1, se presentan los valores promedios de la humedad del suelo para cada una de las épocas. Los resultados mostraron mayor disponibilidad de agua en el suelo en la época húmeda, con $25,32 \%$. Con relación a los sistemas agroforestales, el sistema con abarco presentó mayor contenido de humedad en las dos épocas posiblemente porque esta es una especie de menor extracción.

\section{Radiación fotosintéticamente activa (PAR)}

Los análisis de varianza realizados a la PAR que llega al dosel del $T$. cacao en los diferentes sistemas de sombrío, no detectaron diferencias entre las especies. 
Tabla 1. Humedad del suelo (\%) en época húmeda y seca en dos sistemas agroforestales de cacao en Santander (Colombia).

\begin{tabular}{|l|c|c|c|}
\hline \multicolumn{1}{|c|}{ Localidad } & Tipo sombrío & Época seca & Época húmeda \\
\hline \multirow{2}{*}{ El Carmen } & C. pyriformis & 18,00 & 25,00 \\
\cline { 2 - 4 } & H. brasiliensis & 15,50 & 24,00 \\
\hline \multirow{2}{*}{ Rionegro } & C. pyriformis & 20,50 & 26,80 \\
\cline { 2 - 4 } & T. grandis & 19,50 & 25,50 \\
\hline Media & & 18,37 & 25,32 \\
\hline Coeficiente de variación (\%) & 7,18 & 8,20 \\
\hline Desviación estándar & 1,82 & 2,06 \\
\hline
\end{tabular}

Esto indica que los sistemas de sombrío presentan similar capacidad de proveer sombra al cacao. En El Carmen el sombrío H. brasiliensis permitió una radiación de $570,6 \mu$ moles $\mathrm{m}^{-2} \mathrm{~s}^{-1}$ de fotones en el dosel del T. cacao, mientras que C. pyriformis permitió una PAR de 489,46 $\mu$ moles $\mathrm{m}^{-2} \mathrm{~s}^{-1}$ de fotones. En Rionegro, $C$. pyriformis permitió un nivel de radiación de 697,74 $\mu$ moles $\mathrm{m}^{-2} \mathrm{~s}^{-1}$ de fotones mientras T. grandis permitió $586,71 \mu$ moles $\mathrm{m}^{-2} \mathrm{~s}^{-1}$ de fotones. Los anteriores niveles de PAR son adecuados para el cultivo de T. cacao e indica que los sombríos evaluados proveen un nivel de sombra aproximado que oscila entre el 60 y $70 \%$, tomando como base la radiación PAR en un día soleado en Santander (1.800 $\mu$ moles $\mathrm{m}^{-2} \mathrm{~s}^{-1}$ de fotones). Estos valores que están acordes a lo mencionado por Jaimez et al. (2008) y Quiroz (2010), donde recomiendan que el nivel de sombrío para el cultivo del cacao debe estar entre 50 y $70 \%$ para que las plantas logren mayores tasas fotosintéticas, según las evaluaciones en cacao en el Ecuador y Venezuela. Sin embargo, es indispensable tener en cuenta las condiciones climáticas de cada zona donde se establece el cultivo.

Al comparar las épocas húmeda y seca, en relación con la $P A R$ proveída por los sombríos, se encontraron diferencias entre las especies (Fig. 1). La radiación incidente sobre las plantas de T. cacao fue superior en la época seca, especialmente cuando se usa el sombrío con $H$. brasiliensis. Lo anterior se debe a una mayor radiación incidente en la época de verano, sumado al deshoje natural de los árboles de sombrío durante la época seca, especialmente en $H$. brasiliensis (Rivano, 2015). En El Carmen, el sombrío con C. pyriformis provee niveles de PAR sobre el dosel de T. cacao, que aumentan de 476,53 $\mu$ moles $\mathrm{m}^{-2} \mathrm{~s}^{-1}$ en época húmeda a $502,38 \mu$ moles $\mathrm{m}^{-2} \mathrm{~s}^{-1}$ en época seca, representando un incremento de $5,4 \%$. Para el caso del sombrío con $H$. brasilensis la radiación PAR pasa de 456,25 $\mu$ moles $\mathrm{m}^{-2} \mathrm{~s}^{-1}$ en época húmeda a $684,96 \mu$ moles $\mathrm{m}^{-2} \mathrm{~s}^{-1}$

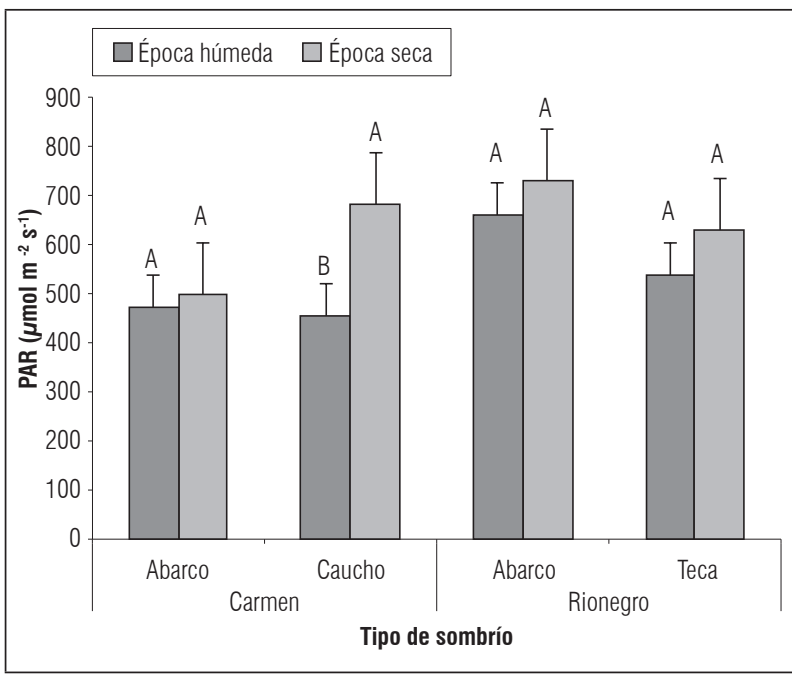

Figura 1. Radiación fotosintéticamente activa (PAR) incidente sobre el dosel de clones de cacao bajo diferentes sombríos en época seca y húmeda en las localidades de El Carmen y Rionegro, Santander (Colombia). Las barras sobre columnas corresponden a error estándar $(n=36)$. Promedios con letras distintas indican diferencia significativa según la prueba de Tukey $(P \leq 0,05)$.

en época seca, representando un aumento del 50\%. Por su parte en Rionegro, el sombrío con C. pyriformis presenta radiación $P A R$ incidente sobre el dosel del T. cacao de $662,9 \mu$ moles $\mathrm{m}^{-2} \mathrm{~s}^{-1}$ en época húmeda y $732,57 \mu$ moles $\mathrm{m}^{-2} \mathrm{~s}^{-1}$ en apoca seca, lo cual representa un incremento del $10,5 \%$. En T. grandis, la radiación pasa de $540,3 \mu$ moles $\mathrm{m}^{-2} \mathrm{~s}^{-1}$ en época húmeda a $633,11 \mu$ moles $\mathrm{m}^{-2} \mathrm{~s}^{-1}$ en época seca, lo que representa un incremento del 17,2\%. Estos resultados permiten concluir que el deshoje natural que ocurre en las especies arbóreas usadas como sombrío permanente en cultivos de T. cacao, durante la época seca inciden en los niveles de radiación sobre el cultivo, encontrando 
en este caso que la especie $H$. brasiliensis, dado su gran deshoje natural en época de verano, presenta los más grandes incrementos en la radiación incidente. Esto limitaría el uso de esta especie como sombrío permanente del cultivo de cacao en zonas donde la época seca es muy drástica o prolongada. T. grandis por su parte, presenta deshoje natural en la época seca en menor proporción, por lo tanto, esta especie se puede utilizar como sombrío permanente para cacao en zonas con épocas secas poco prolongadas. Por el contrario, se observan las bondades de la especie C. pyriformis, quién presenta menor porcentaje de deshoje natural en la época seca, lo cual permite mantener niveles de radiación adecuados para el cultivo de cacao durante todo el año.

\section{Tasa de fotosíntesis neta $(A)$}

\section{Efecto de los sistemas agroforestales}

Con relación a los sistemas agroforestales, se detectaron efectos de los sistemas de sombrío $(P \leq 0,05)$, específicamente en la localidad de Rionegro. En este caso, se presentó mayor $A$ en los clones establecidos bajo el sombrío con C. pyriformis, con valores de 5,39 $\mu$ moles $\mathrm{CO}_{2} \mathrm{~m}^{-2} \mathrm{~s}^{-1}$ comparada con tasas de 5,05 $\mu$ moles $\mathrm{CO}_{2} \mathrm{~m}^{-2} \mathrm{~s}^{-1}$ en los clones bajo sombrío con T. grandis. En El Carmen, no se detectaron efectos significativos $(P>0,05)$, del sistema de sombrío, sin embargo, la tendencia indica que $A$ del $T$. cacao son mayores bajo sombrío con C. pyriformis que con $H$. brasiliensis $\left(4,75\right.$ y $4,57 \mu$ moles $\mathrm{CO}_{2} \mathrm{~m}^{-2} \mathrm{~s}^{-1}$, respectivamente) (Fig. 2).

\section{Efecto de la época y el sistema agroforestal}

Considerando la época del año, se encontró que $A$ se reducen en la época seca en El Carmen, pero no en Rionegro (Fig. 3). En efecto, en El Carmen las tasas de A pasaron de 4,94 $\mu$ moles $\mathrm{CO}_{2} \mathrm{~m}^{-2} \mathrm{~s}^{-1}$ en época húmeda a 4,38 $\mu$ moles $\mathrm{CO}_{2} \mathrm{~m}^{-2} \mathrm{~s}^{-1}$ en época seca. Por su parte en Rionegro las tasas de fotosíntesis se mantienen muy similares tanto en época húmeda 5,32 $\mu$ moles $\mathrm{CO}_{2} \mathrm{~m}^{-2} \mathrm{~s}^{-1}$ como en época seca $5,12 \mu$ moles $\mathrm{CO}_{2} \mathrm{~m}^{-2} \mathrm{~s}^{-1}$ (Fig. 3). Aunque durante la época seca en esta última localidad se redujo sustancialmente, probablemente los sombríos utilizados en Rionegro al presentar un menor deshoje, brindaron un mayor sombrío al T. cacao y permiten mantener una mayor disponibilidad de humedad en el suelo durante la época seca, lo que permite mantener la actividad fisiológica de los mismos.
Estos resultados concuerdan con los reportados por Joly y Hahn (1989); Tezara et al. (2008), Pereyra et al. (2007) y Cao et al. (2009), donde se determina que la A se disminuye en las épocas secas, debido al cierre

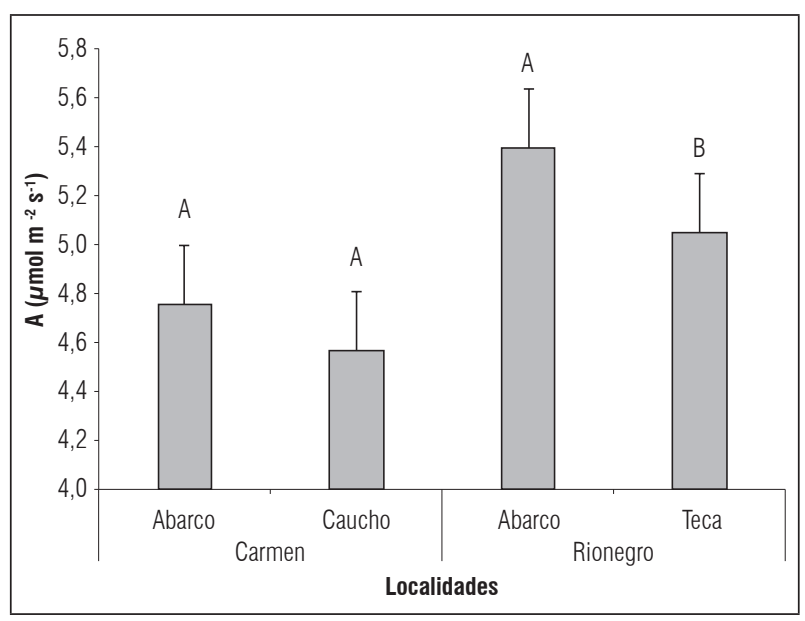

Figura 2. Efecto del sombrío sobre la tasa fotosintética neta (A) de clones de cacao en las localidades de EI Carmen y Rionegro, Santander (Colombia). Las barras sobre columnas corresponden al error estándar $(n=72)$. Promedios con letras distintas indican diferencia significativa según la prueba de Tukey $(P \leq 0,05)$.

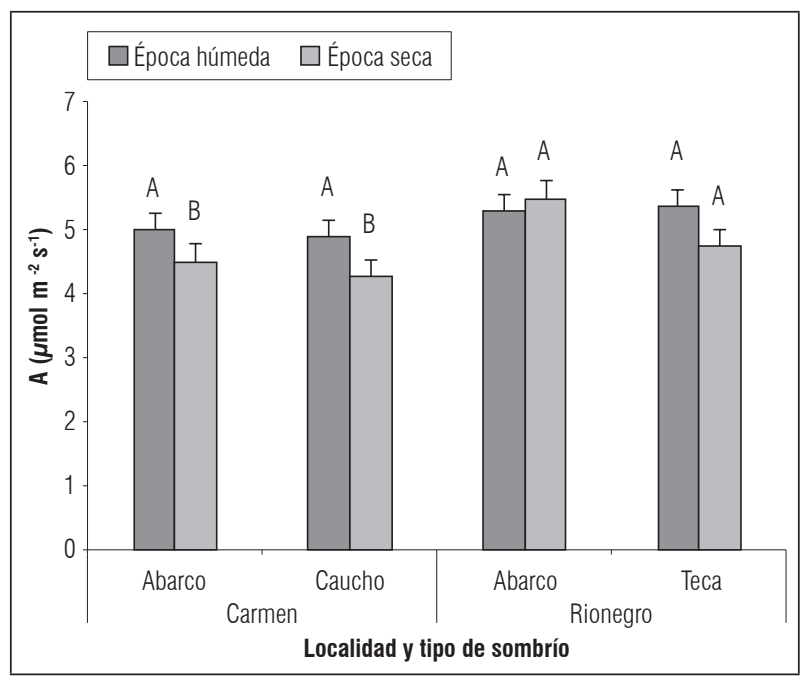

Figura 3. Efecto de la época del año sobre la tasa fotosintética neta de clones de cacao en las localidades de El Carmen y Rionegro, Santander (Colombia). Las barras sobre columnas corresponden a error estándar $(n=36)$. Promedios con letras distintas indican diferencia significativa según la prueba de Tukey $(P \leq 0,05)$. 
parcial de las estomas y a la disminución de la transpiración e intercambio gaseoso en las hojas de las plantas (Taiz y Zeiger, 2010; García, 2014). Por otra parte, la resistencia estomática $\left(g_{s}\right)$ en El Carmen pasó de $80,69 \mathrm{mmol} \mathrm{m}^{-2} \mathrm{~s}^{-1}$ en época húmeda a 73,19 $\mathrm{mmol} \mathrm{m}^{-2}$ $\mathrm{s}^{-1}$ en época seca, en Rionegro, pasó de 100,69 mmol $\mathrm{m}^{-2} \mathrm{~s}^{-1}$ en época húmeda a $94,84 \mathrm{mmol} \mathrm{m}^{-2} \mathrm{~s}^{-1}$ en época seca, lo que demuestra el cierre parcial de estomas y mayor resistencia al flujo de $\mathrm{CO}_{2}$ en los clones durante los periodos de verano.
Con relación al comportamiento individual de los clones, el desempeño $A$ no tiene efectos significativos en las dos localidades. Se encontró que, en El Carmen durante la época húmeda, el clon TCS19 presenta la mayor A con 5,63 $\mu$ moles $\mathrm{CO}_{2} \mathrm{~m}^{-2} \mathrm{~s}^{-1}$. En Rionegro, localidad menos afectada por la baja precipitación en la época seca, igualmente el clon TCS19 presenta las mayores tasas de fotosíntesis, tanto en época húmeda como en época seca, con valores de 5,38 y 5,95 $\mu$ moles $\mathrm{CO}_{2} \mathrm{~m}^{-2} \mathrm{~s}^{-1}$, respectivamente (Figs. 4 y 5 ).

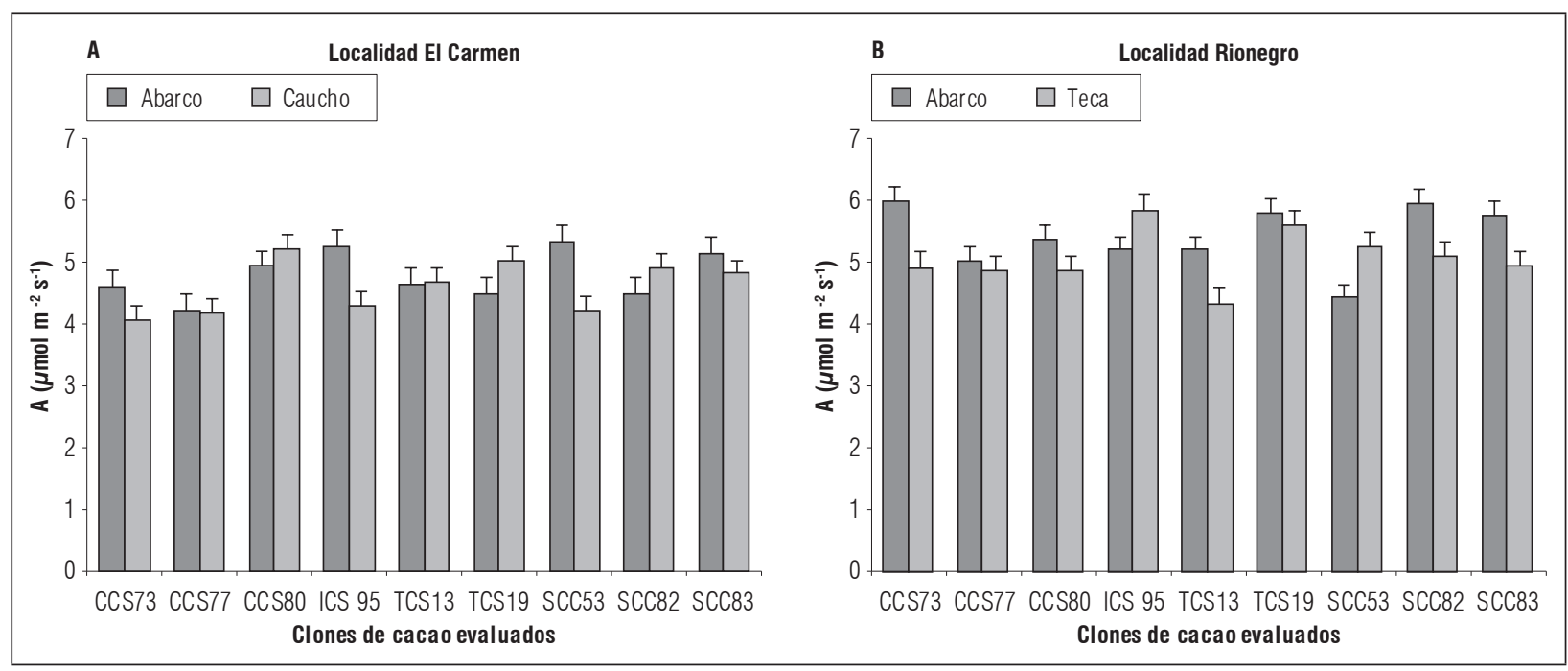

Figura 4. Efecto del sistema de sombrío sobre la tasa fotosintética neta $(\boldsymbol{A})$ de clones de cacao en las localidades de El Carmen y Rionegro, Santander (Colombia). Las barras sobre columnas corresponden a error estándar $(n=8)$.

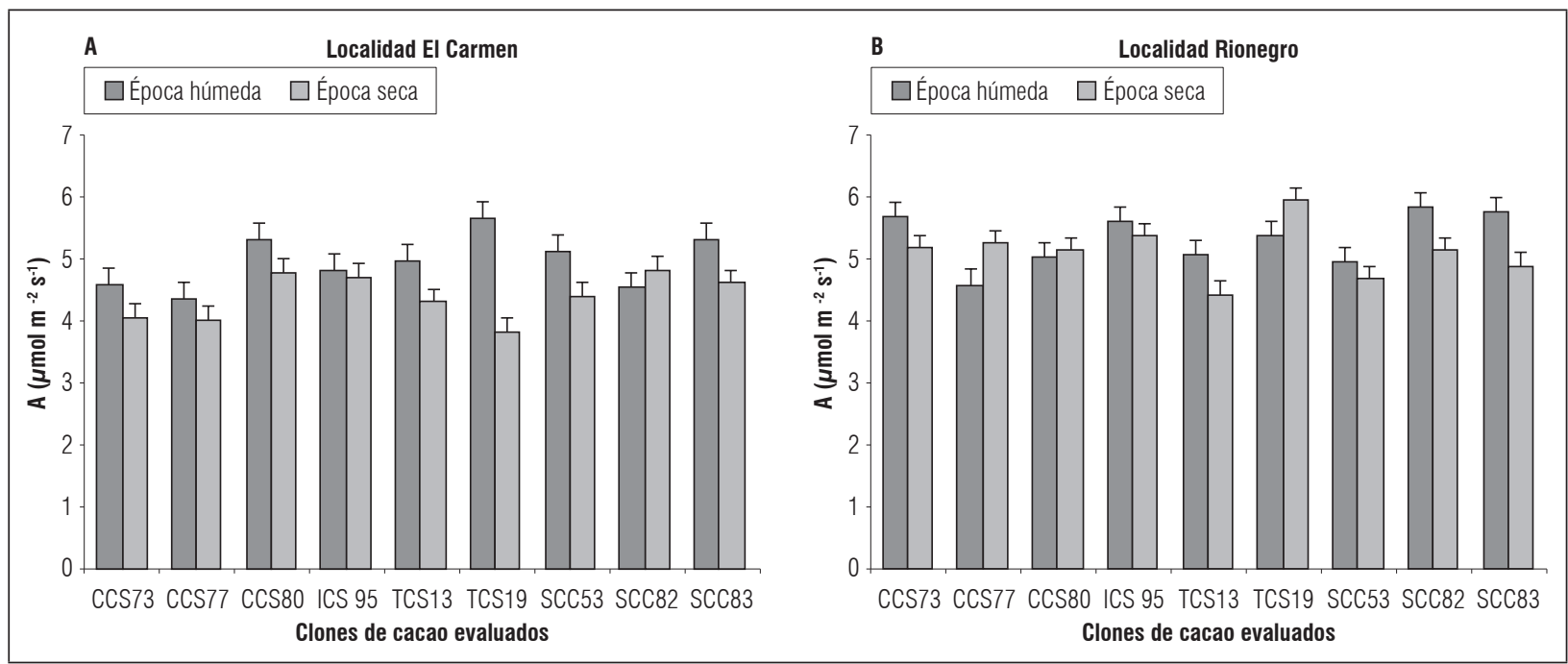

Figura 5. Efecto de la época sobre la tasa fotosintética de los clones de cacao en las localidades de El Carmen y Rionegro, Santander (Colombia). Las barras sobre columnas corresponden a error estándar $(n=8)$. 
Estos estudios permiten concluir que la $A$ de los clones de cacao, bajo los sombríos con C. pyriformis, $T$. grandis y $H$. brasiliensis, se ajusta a los valores considerados adecuados para este cultivo. De hecho, las tasas de $A$ encontrados en estos experimentos fueron superiores a las reportadas en los estudios realizados por Jaimez et al. (2008) y Villa (2016). Es de resaltar a C. pyriformis dentro de estos sistemas de sombrío, por presentar las mayores tasas de $A$ en los clones bajo este sombrío, lo que podría redundar en mayor disponibilidad de fotoasimilados para las plantas de T. cacao y por ende en mayor capacidad productiva (Ribeiro et al., 2008).

La fotosíntesis en $T$. cacao responde de manera diferencial a los niveles de luz presentes en los sistemas y a la genética de cada uno de los cultivares que conllevan a respuestas en los aparatos fotosintéticos (Taiz y Zeiger, 2010). En general mayores tasas fotosintéticas están asociadas a mayor capacidad de asimilación de $\mathrm{CO}_{2}$, mayor concentración de la clorofila, mayores niveles de nitrógeno y proteínas en las hojas, mayor actividad de la Rubisco, mayor desarrollo de los cloroplastos y mejor metabolismo en la planta (Ribeiro et al., 2008).

Los valores promedios A registrados fueron superiores a los reportados por otros autores para cacao. Por ejemplo, Jaimez et al. (2008), en una evaluación de 53 accesiones de T. cacao en el estado de Zulia en Venezuela, reportan valores de $A$ entre 4,0 y $4,4 \mu$ moles $\mathrm{CO}_{2} \mathrm{~m}^{-2} \mathrm{~s}^{-1}$; estudios recientes en Colombia reportan valores de $A$ entre 3,6 y 18,1 $\mu$ moles $\mathrm{CO}_{2} \mathrm{~m}^{-2} \mathrm{~s}^{-1}$ que varían con el manejo de la copa y la posición de las ramas y hojas en las plantas de cacao (Villa, 2016).

\section{Transpiración}

\section{Efecto del sistema agroforestal}

El sistema de sombrío no tuvo efectos sobre la tasa de transpiración ( $E$ ) en Rionegro, pero si en El Carmen, donde el tipo de sombrío utilizado tuvo efectos significativos sobre las tasas de $E$ registradas en los clones de cacao. En esta localidad, los clones presentaron mayores tasas transpiratorias bajo el sombrío con $C$. pyriformis, con valores de $2,15 \mathrm{mmol} \mathrm{H}_{2} 0 \mathrm{~m}^{-2} \mathrm{~s}^{-1}$, mientras que los clones bajo sombrío $H$. brasiliensis, las tasas de $E$ fueron de $1,91 \mathrm{mmol} \mathrm{H}_{2} 0 \mathrm{~m}^{-2} \mathrm{~s}^{-1}$ (Fig. 6). Esto indica una mejor condición de humedad en el suelo y menor cierre parcial de estomas para los clones de $T$. cacao bajo el sistema de sombrío con C. pyriformis. Lo que permite mantener mayor actividad de intercambio gaseoso, tanto fotosíntesis como transpiración.
En Rionegro, no se detectaron efectos de los sistemas de sombrío sobre la $E$ para los clones de T. cacao. En esta localidad, los valores de $\mathrm{E}$ registrados en los clones fueron de 2,29 y $2,25 \mathrm{mmol} \mathrm{H}_{2} 0 \mathrm{~m}^{-2} \mathrm{~s}^{-1}$, bajo el sombrío con C. pyriformis y T. grandis, respectivamente (Fig. 6).

\section{Efecto de la época y el sistema agroforestal}

Con relación a la época de medición, los resultados mostraron que los clones registran menores tasas de transpiración en la época seca debido a la baja disponibilidad de agua en el suelo. Con relación al sistema de sombrío, en El Carmen, la transpiración más baja se observó en los clones de T. cacao bajo el sombrío con C. pyriformis, indicando que este sistema brinda mejor condición a los clones de T. cacao. Por el contrario, en esta misma localidad se registra mayor $E$ en época seca, con el sombrío con $H$. brasiliensis.

En Rionegro, se mantienen la $E$ del T. cacao en época húmeda y seca, bajo los dos sistemas de sombrío ( $C$. pyriformis y $T$. grandis), indicando la protección que brindan estos dos sistemas en épocas en que la humedad del suelo no es muy alta. Sin embargo, es de destacar que en esta localidad la precipitación es alta y el periodo seco es más corto (Fig. 6).

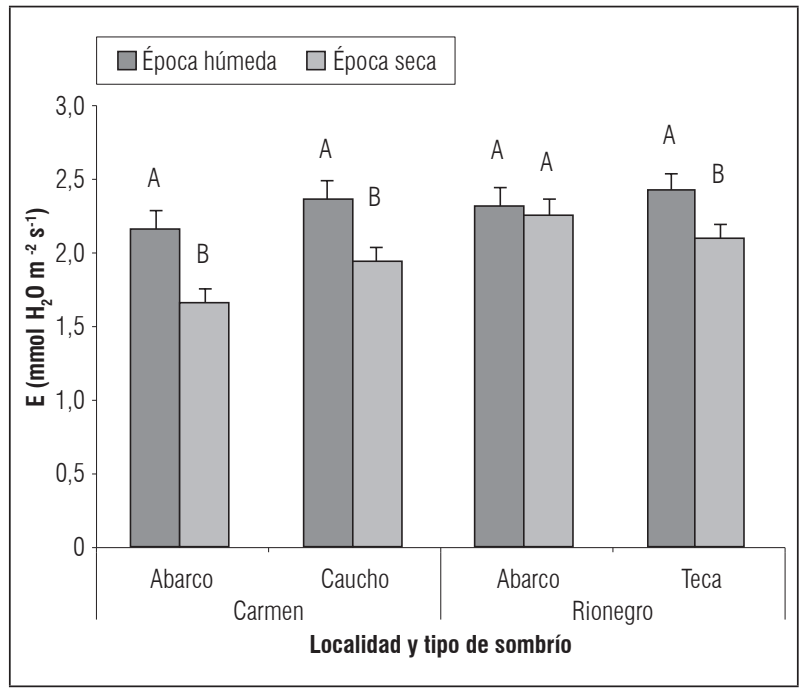

Figura 6. Efecto del sombrío y la época sobre las tasas de transpiración en clones de cacao en las localidades de El Carmen y Rionegro, Santander (Colombia). Las barras sobre columnas corresponden a error estándar $(n=36)$. Promedios con letras distintas indican diferencia significativa según la prueba de Tukey $(P \leq 0,05)$. 
Individualmente, entre los clones no se presentan efectos significativos en la $E$, los clones presentan valores de 1,94 y $2,35 \mathrm{mmol} \mathrm{H}_{2} \mathrm{O} \mathrm{m}^{-2} \mathrm{~s}^{-1}$ para el SCC53 y SCC73 en Rionegro. En El Carmen, los clones presentan valores de $E$ entre 1,88 y $2,36 \mathrm{mmol} \mathrm{H}_{2} 0 \mathrm{~m}^{-2} \mathrm{~s}^{-1}$ para el TCS19 y SCC83 (Fig. 7).

Los resultados obtenidos son similares a estudios realizados por García (2014) y Tezara (2008), donde obtuvieron valores más bajos de $E$ en la época seca (Fig. 6) o bajo condiciones de estrés hídrico, en la cual mencionan que el sistema de sombrío ejerce un efecto.

La transpiración es menor en la localidad de El Carmen, debido a la menor disponibilidad de agua en el suelo, lo cual provoca estrés hídrico en las plantas de cacao (Fig. 6), relacionado con el cierre parcial de estomas, generado por la disminución de la conductancia. En esta localidad, la resistencia estomática en los genotipos SCC83 y SCC82 registraron 91,41 y 65,16 mmol m $\mathrm{m}^{-2}$, respectivamente.

En Rionegro no se presentan diferencias entre los clones, sin embargo, el SCC83 registró mayor $g_{s}$ con $118,75 \mathrm{mmol} \mathrm{m}^{-2} \mathrm{~s}^{-1}$ y el menor valor el CCS80 con $86,56 \mathrm{mmol} \mathrm{m}^{-2} \mathrm{~s}^{-1}$. Los clones presentan mayor resistencia estomática en la localidad de Rionegro, esto se atribuye a la mayor disponibilidad de agua en el suelo en la época seca y la época de lluvias, lo cual provoca disminución de estrés hídrico en la planta y mejora la apertura de estomas.

Los resultados demuestran que la menor apertura estomática es causada por la disminución de la gs es la época seca, donde el suelo presenta menor disponibilidad de agua, conllevando a disminuir la transpiración y la asimilación de $\mathrm{CO}_{2}$ en las plantas de T. cacao. Esto afecta el crecimiento y producción especialmente en la fase de establecimiento del cultivo cuando las plantas son más vulnerables al estrés hídrico (Araque et al., 2012).

Lo anterior es concordante con estudios realizados por Jaimez et al. (2008), donde se determinó que las accesiones de cacao en periodo de lluvias presentan mayor gs que en épocas secas, de igual manera determina que el sitio y la sombra presente alrededor de las plantas afectan factores micro climáticos como la temperatura, humedad relativa, disponibilidad de agua y la fertilidad del suelo, que tienen gran influencia en la actividad fisiológica de las plantas.

De igual manera Rada et al. (2005) realizaron estudios en plantas de 4 años de edad, donde la $g_{s}$ disminuyó en plantas sometidas a condiciones de estrés hídrico. Esto es una estrategia que tienen las plantas para reducir la pérdida de agua atribuible al ajuste osmótico que realizan las células, teniendo en cuenta que la planta de T. cacao es C3, susceptible a la deshidratación (DaMatta, 2003; Daymond et al., 2011).

\section{Uso eficiente del agua (EUA)}

No se encontraron diferencias en el Uso Eficiente del Agua $(U E A)$ de los clones de T. cacao por efecto de las épocas, tipos de sombrío o su interacción. Esto indica que los sistemas de sombrío evaluados en estos

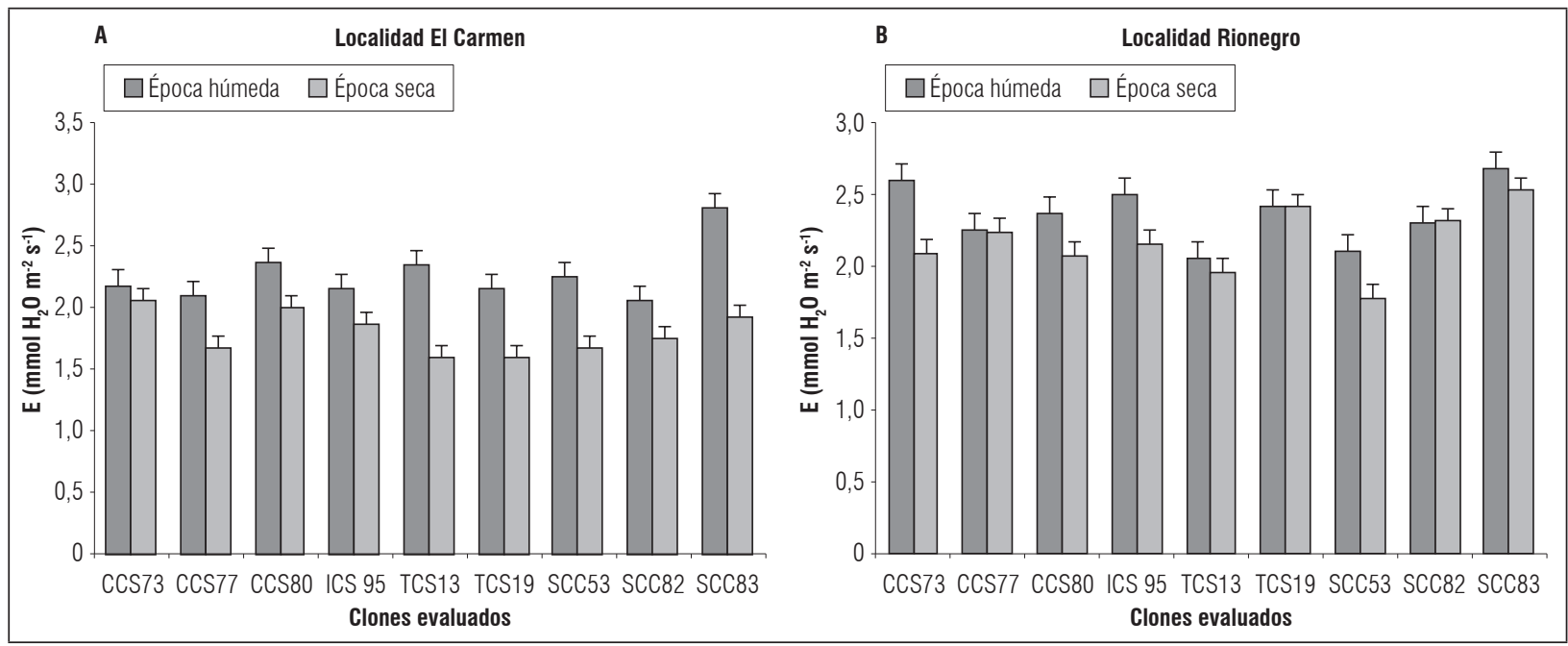

Figura 7. Efecto de la época sobre las tasas de transpiración en clones de cacao en las localidades de El Carmen y Rionegro, Santander (Colombia). Las barras sobre columnas corresponden al error estándar $(n=8)$. 
ensayos permiten atenuar los efectos de la falta de agua en las épocas secas del año, manteniendo las actividades fisiológicas de las plantas. En cuanto a los clones, presentaron similares eficiencias entre sí (Tab. 2).

Estos resultados concuerdan con lo reportado por Tezara et al. (2009), quienes encontraron que entre las épocas de lluvia y sequia no se presentan diferencias significativas en EUA. Sin embargo, estos autores lograron registrar variabilidad entre los cultivares. De igual manera estudios realizados por García (2014), encontraron que las tasas de EUA en plantas sometidas a condiciones de estrés hídrico se incrementaban a medida que aumentaban los niveles de estrés.

En este caso, los clones de cacao TCS13 y TCS19 presentan tendencias mayores en las tasas de EUA en las épocas secas (Tab. 2), además presentan tasas fotosintéticas similares a los demás clones evaluados, por tanto, se constituyen en genotipos de importancia agronómica. Es necesario correlacionar estas variables fisiológicas con el rendimiento productivo para obtener recomendaciones de su uso en las diferentes regiones productoras (Tezara et al., 2008).

\section{CONCLUSIONES}

El tipo de sombrío utilizado en los sistemas agroforestales tiene influencia sobre el comportamiento ecofisiológico de los clones de T. cacao, los mayores efectos del sombrío se ejercieron sobre las tasas de fotosíntesis. El sombrío con C. pyriformis presentó mayores ventajas que con $H$. brasiliensis y $T$. grandis. Los beneficios de esta especie pueden ser observados en época húmeda y seca, lo que permite mantener la actividad fisiológica del cacao durante todo el año. Esto permite recomendar la inclusión de esta especie como sombrío para siembras nuevas de cacao en Santander.

En cuanto a los clones de T. cacao, las mayores ventajas se observaron en el TCS13 y TCS19, presentan mayor tasa de EUA en las épocas secas, altas tasas fotosintéticas y conductancia estomática, por lo que se constituyen en clones de importancia agronómica. Es necesario correlacionar estas variables con el rendimiento para obtener recomendaciones de su uso en las diferentes regiones productoras.

Tabla 2. Tasa de fotosíntesis neta $(A)$, conductancia estomática $\left(g_{s}\right)$ y eficiencia en el uso de agua $(E U A)$ en genotipos de cacao plantados en Santander, Colombia.

\begin{tabular}{|c|c|c|c|c|}
\hline Clon & Época & $\begin{array}{c}A \\
\left(\mu \mathrm{mol} \mathrm{m} \mathrm{m}^{-2} \mathrm{~s}^{-1}\right) \\
\end{array}$ & $\begin{array}{c}g_{s} \\
\left(\mathrm{~mol} \mathrm{~m}^{-2} \mathrm{~s}^{-1}\right) \\
\end{array}$ & $\begin{array}{c}\text { EUA } \\
\left(\mu \mathrm{mol} \mathrm{CO} / \mathrm{mmol} \mathrm{H}_{2} \mathrm{O}\right)\end{array}$ \\
\hline \multirow{2}{*}{ CCS73 } & Húmeda & 5,125 & 88,13 & 2,231 \\
\hline & Seca & 4,616 & 85,78 & 2,402 \\
\hline \multirow{2}{*}{ CCS77 } & Húmeda & 4,469 & 85,31 & 2,103 \\
\hline & Seca & 4,626 & 80,93 & 2,623 \\
\hline \multirow{2}{*}{ CCS80 } & Húmeda & 5,168 & 85,63 & 2,332 \\
\hline & Seca & 4,96 & 79,84 & 2,609 \\
\hline \multirow{2}{*}{ ICS 95} & Húmeda & 5,197 & 94,06 & 2,396 \\
\hline & Seca & 5,04 & 87,19 & 2,542 \\
\hline \multirow{2}{*}{ TCS13 } & Húmeda & 5,007 & 91,56 & 2,34 \\
\hline & Seca & 4,363 & 80,34 & 2,899 \\
\hline \multirow{2}{*}{ TCS19 } & Húmeda & 5,504 & 94,53 & 2,638 \\
\hline & Seca & 4,881 & 80,63 & 2,669 \\
\hline \multirow{2}{*}{ SCC53 } & Húmeda & 5,022 & 90,31 & 2,392 \\
\hline & Seca & 4,527 & 81,44 & 2,592 \\
\hline \multirow{2}{*}{ SCC82 } & Húmeda & 5,179 & 83,13 & 2,451 \\
\hline & Seca & 4,98 & 81,25 & 2,562 \\
\hline \multirow{2}{*}{ SCC83 } & Húmeda & 5,534 & 103,59 & 2,14 \\
\hline & Seca & 4,74 & 98,75 & 2,465 \\
\hline \multicolumn{2}{|l|}{ Media } & 4,94 & 87,36 & 2,47 \\
\hline \multicolumn{2}{|c|}{ Coeficiente de variación (\%) } & 29,06 & 26,33 & 43,06 \\
\hline \multicolumn{2}{|c|}{ Desviación estándar } & 1,44 & 22,99 & 1,09 \\
\hline
\end{tabular}




\section{AGRADECIMIENTOS}

Los autores agradecen a la Corporación Colombiana de Investigación Agropecuaria (Corpoica) por suministrar los equipos y recursos necesarios para el registro de los datos en campo. Igualmente, a Eduar Antolinez y Eliana Báez por el apoyo en el registro de la información.

Conflicto de intereses: el manuscrito fue preparado y revisado con la participación de los autores, quienes declaran no tener algún conflicto de interés que coloquen en riesgo la validez de los resultados aquí presentados.

\section{REFERENCIAS BIBLIOGRÁFICAS}

Almeida, A.A.F.D. y R.R. Valle. 2007. Ecophysiology of the cacao tree. Braz. J. Plant Physiol. 19(4), 425-448. Doi: 10.1590/S1677-04202007000400011

Araque, O., R.E. Jaimez, W. Tezara, I. Coronel, R. Urich y W. Espinoza. 2012. Comparative photosynthesis, water relations, growth and survival rates in juvenile Criollo cacao cultivars (Theobroma cacao) during dry and wet seasons. Exp. Agric. 48(4), 513-522. Doi: 10.1017/ S0014479712000427

Arguello, O., L.A. Mejía, N. Contreras y J.A. Toloza. 1999. Manual de caracterización morfoagronómica de clones élite de cacao (Theobroma cacao L.) en el nororiente colombiano. Corporación Colombiana de Investigación Agropecuaria (Corpoica), Bucaramanga, Colombia.

Cao, J., E. Boone, M. Aguilar, P. Siqueira, S. Souza, F. Folli y D. Pinto. 2009. Physiological characteristics and growth of grafts of Theobroma cacao L. bred under different doses of water retaining polymers and submitted to a cycle of moisture deficit. pp. 341-359. En: $16^{\text {th }}$ International coca Research Conference. Towards rational cocoa production and efficient use for a sustainable world cocoa economy. Denpasar, Bali, Indonesia.

DaMatta, F.M. 2003. Drought as a multidimensional stress affecting photosynthesis in tropical tree crops. pp. 227-265. En: Hemantaranjan, A. (ed.). Advances in plant physiology. Vol. 5. Scientific Publishers, Jodhpur, India.

Daymond, A.J., P.J. Tricker y P. Hadley. 2011. Genotypic variation in photosynthesis in cacao is correlated with stomatal conductance and leaf nitrogen. Biol. Plant, 55(1), 99-104. Doi: 10.1007/s10535-011-0013-y

Fedecacao. 2017. Estadísticas y recaudo. En: Federación Nacional de Cacaoteros y Fondo Nacional del Cacao. http://www.fedecacao.com.co/portal/index.php/ es/2015-02-12-17-20-59/nacionales; consulta: agosto de 2017.

García, J. 2014. Caracterización de las respuestas fisiológicas y bioquímicas en tres clones de cacao (Theobroma cacao L.) sometidos a diferentes niveles de déficit hídrico. Tesis de doctorado. Escuela de Posgrados, Facultad de Ciencias Agrarias, Universidad Nacional de Colombia, Bogotá.

Jaimez, R.E., W. Teraza, I. Coronel y R. Urich. 2008. Ecofisiología del cacao (Theobroma cacao): su manejo en el sistema agroforestal. Sugerencias para su mejoramiento en Venezuela. Rev. For. Venez. 52(2), 253-258.

Joly, R.J. y D.T. Hahn. 1989. Net $\mathrm{CO}_{2}$ assimilation of cacao seedlings during periods of plant water deficit. Photosyn. Res. 21(3), 151-159. Doi: 10.1007/BF00037179

Mejía, L.A. y G.E. Palencia. 2004. Una aproximación a los sistemas agroforestales con cacao. Corpoica, Bucaramanga, Colombia.

Pereyra, G., V. Villalobos, O. Rondón, I. Chacón, R. Jaimez y W. Tezara. 2007. Intercambio gaseoso en diferentes cultivares de cacao (Theobroma cacao L.). pp. 669-672. En: XVII Congreso de Botánica de Venezuela. Maracaibo, Venezuela.

Quiroz, J. 2010. Sistemas de sombra de cacao con maderables. Boletín técnico No. 151. Instituto de Nacional de Investigaciones Agropecuarias (INIAP), cantón Yaguachi, Ecuador.

Rada, F., R.E. Jaimez, C. García-Núñez, A. Azócar y M.E. Ramírez. 2005. Relaciones hídricas e intercambio de gases en Theobroma cacao var. Guasare bajo períodos de déficit hídrico. Rev. Fac. Agron. (LUZ) 22, 112-120.

Ribeiro, M.A.O., J.O. da Silva, W.M. Aitken, R.C.R. Machado y V.C. Baligar. 2008. Nitrogen use efficiency in cacao genotypes. J. Plant Nutr. 31(2), 239-249. Doi: 10.1080/01904160701853720

Rivano, F. 2015. Adaptación de clones de caucho en América latina, en zonas de Microcyclus ulei y zonas de escape. Workshop Cirad-Agritrop, Medellín, Colombia.

Taiz, L. y E. Zeiger. 2010. Plant physiology. 5a ed. Sinauer Associates, Sunderland, MA, USA.

Tezara, W., G. Pereyra, O. Marín, V. Villalobos, S. Irazábal, A. González, R. Colombo, R. Urich, I. Coronel y A. Herrera 2008. Variación interespecífica en la respuesta fotosintética en diferentes formas de vida en zonas áridas y eficiencia de uso de agua en 32 accesiones de cacao. pp. 233-236. En: Memorias del Instituto de Biología Experimental. Facultad de Ciencias, Universidad Central de Venezuela, Caracas, Venezuela.

Tezara, W., I. Coronel, R. Urich, O. Marín, R. Jaimez y I. Chacon 2009. Plasticidad ecofisiológica de árboles de Cacao (Theobroma cacao L.) en diferentes ambientes de Venezuela. p. 5. En: Memorias III Congreso Latinoamericano y Caribeño de Estudiantes, São Lourenço-MG, Brasil.

Villa, M.R. 2016. Dinámica del crecimiento y desempeño en el intercambio de gases del cacao como respuesta al manejo del dosel. Tesis de maestría. Facultad de Ciencias Agropecuarias, Universidad Nacional de Colombia, Medellín, Colombia. 